T. Kawada

Nagoya Math. J.

Vol. 35 (1969), 109-132

\title{
ON THE UPPER AND LOWER GLASS FOR GAUSSIAN PROGESSES WITH SEVERAL PARAMETERS
}

\author{
TAKAYUKI KAWADA
}

1. In the study on Hölder-continuity of Brownian motion, A.N.Kolmogorov introduced the concept of upper and lower classes and presented a criterion with the integral form to test whether some function belongs to upper or lower class; the so-called Kolmogorov's test (I.Petrovesky [10]). P.Lévy considered the upper and lower class with regard to the uniform continuity of Brownian motion. We shall recall the definition of the upper and lower classes. We shall call $\varphi(t)$ a function belonging to the upper class with regard to the uniform continuity of Brownian motion $x(t)$ if there exists a positive number $\varepsilon(w)$ such that, for almost all $w$,

$$
\begin{gathered}
\left|t-t^{\prime}\right| \leqq \varepsilon(w) \quad \text { implies } \\
\left|x(t)-x\left(t^{\prime}\right)\right| \leqq\left|t-t^{\prime}\right|^{1 / 2} \cdot \varphi\left(1 /\left|t-t^{\prime}\right|\right) .
\end{gathered}
$$

On the otherhand, we shall call $\varphi(t)$ a function belonging to the lower class with regard to the uniform continuity of Brownian motion $x(t)$ if, for almost all $w$ and for any positive number $\delta$, there exist a pair $\left(t, t^{\prime}\right)$ such that $\left|t-t^{\prime}\right| \leqq \delta$ and (1. 1) does not hold.*2

P.Lévy showed that the function

$$
\varphi(t)=c \cdot(2 \log t)^{1 / 2}
$$

belongs to the upper class if $c>1$ and to the lower class if $c<1$ (P. Lévy [8]). Further, K.L.Chung, P.Erdös and T.Sirao [3] proved that a continuous, non-negative and non-decreasing function $\varphi(t)$ belongs to upper or lower class according as the integral

Received October 1, 1968

*) It turns out that every 'continuous positive and non-decreasing function belongs to either upper class or lower class. 


$$
\int^{\infty} \varphi^{3}(t) \cdot \exp \left(-\frac{1}{2} \varphi^{2}(t)\right) d t
$$

is convergent or divergent. T.Sirao [11] extended these results to the case of Brownian motion with several parameters of P.Lévy (P.Lévy [9]). Recently T.Sirao and H.Watanabe [12] have studied Hölder-continuity of a class of Gaussian processes and obtained a similar criterion of upper and lower classes. Their result is an extension of Yu.K. Belayev [1] in some sense.

In this paper we shall try to extend the result of T.Sirao and H.Watanabe [12] to the Gaussian processes with several parameters:

Consider the Gaussian processes $\{x(A, w) ; A \in D\}$ such that

$$
\begin{gathered}
E\{x(A)\}=0, \\
E\{x(A) \cdot x(B)\}=\frac{1}{2}\left\{d^{\alpha}(0, A)+d^{\alpha}(0, B)-d^{\alpha}(A, B)\right\},
\end{gathered}
$$

where

$$
\begin{aligned}
& D=\left\{A=\left(a_{1}, a_{2}, \cdots, a_{N}\right) ; a_{i} \in R,\left|a_{i}\right| \leqq 1, i=1,2, \cdots, N\right. \\
& 0<\alpha \leqq 1, d^{2}(A, B)=\sum_{i=1}^{N}\left(a_{i}-b_{i}\right)^{2} \text { and } 0=(0, \cdots, 0) .
\end{aligned}
$$

The right-hand side of (1.2) becomes a positive definite kernel ( $R$. Gangolli [6]). From (1.2) we have

$$
E\left\{(x(A)-x(B))^{2}\right\}=d^{x}(A, B)
$$

and this condition implies the continuity of almost all sample paths (X. Fernique [5], R.M.Dudley [4]). We define, after P.Lévy and T.Sirao, the upper and lower class for the above Gaussian processes. Set

$$
\sigma^{2}(A, B)=E\left\{(x(A)-x(B))^{2}\right\} .
$$

If there exists a positive number $\varepsilon(w)$ such that, for almost all $w, d(A, B) \leqq \varepsilon(w)$ implies

$$
|x(A)-x(B)| \leqq \sigma(A, B) \cdot \varphi(1 / d(A, B)),
$$

then $\varphi(t)$ is called the function belonging to upper class with regard to the uniform continuity of this process.

On the otherhand if, for almost all $w$ and for any positive number $\delta$, there exists a pair $(A, B)$ such that $d(A, B) \leqq \delta$ and (1.4) does not hold, then 
$\varphi(t)$ is defined as the function belonging to lower class with regard to the uniform continuity of the process. Denoting the upper and lower classes with regard to uniform continuity as $U^{u}$ and $L^{u}$ respectively, we have;

TheOREm. Let $\varphi(t)$ be a positive, continuous and non-decreasing function of $t \in[e, \infty) . \quad$ Set

$$
K[x]=x^{\frac{4 N}{\alpha}-1} \cdot \exp \left(-x^{2} / 2\right)
$$

and

$$
I(\varphi)=\int_{e}^{\infty} t^{N-1} \cdot K[\varphi(t)] d t .
$$

Then

$$
\begin{array}{lll}
\varphi(t) \in U^{u} & \text { if } & I(\varphi)<\infty \\
\varphi(t) \in L^{u} & \text { if } & I(\varphi)=\infty .
\end{array}
$$

and

As the consequence, we have the following:

Corollary. If we set for any positive number $\varepsilon$,

$$
\varphi_{i}(t, \varepsilon)=\left\{2 N \log t+\left(\frac{4 N}{\alpha}+1+(-1)^{i} \cdot \varepsilon\right) \cdot \log \log t\right\}^{1 / 2}, \quad i=1,2,
$$

then

$$
\varphi_{1}(t, \varepsilon) \in L^{u}, \varphi_{2}(t, \varepsilon) \in U^{u} \text { and } \varphi_{1}(t, 0) \in L^{u} .
$$

If we set for any positive number $\varepsilon$ and for any integer $n \geqq 3$,

$$
\begin{aligned}
& \varphi_{j}(t, \varepsilon)= \\
& \left\{2 N \log t+\left(\frac{4 N}{\alpha}+1\right) \cdot \log _{(2)} t+2\left(\log _{(3)} t+\cdots+\log _{(n-1)} t\right)+\left(2+(-1)^{j} \cdot \varepsilon\right) \log _{(n)} t\right\}^{1 / 2}, \\
& j=3,4,
\end{aligned}
$$

where

$$
\log t=\log _{(1)} t, \quad \log \underset{(n)}{t}=\log \left(\log _{(n-1)} t\right),
$$

then

$$
\varphi_{3}(t, \varepsilon) \in L^{u}, \quad \varphi_{4}(t, \varepsilon) \in U^{u} \quad \text { and } \quad \varphi_{3}(t, 0) \in L^{u} .
$$

The contents of the paper are as follows; In section 2, we shall show that it is enough to prove the theorem only for some restricted class of functions, which have, roughly speaking, the same order with $(\log t)^{1 / 2}$. The first half of section 3 is devoted to define the sequence of events and to order them with a numbering. This device is convenient throughout the proof, in particular, in referring Borel-Cantelli lemma and K.L.ChungP.Erdös lemma. In the second half of section 3, Lemma 2 is stated, which is the key lemma in the proof of the theorem. Since many other lemmas 
are necessary for the proof of Lemma 2, it is postponed in section 5 . The proof of theorem will be completed in section 4 .

The author is greatly indebted to Professors T. Sirao and H. Watanabe who proposed the problem with several suggestions and communicated their result [12].

2. Let $F$ be the class of functions:

$$
F=\left\{f(t) ; f_{1}(t) \leqq f(t) \leqq f_{2}(t) \text {, for } t \geqq e\right\}
$$

where

$$
f_{1}(t)=\left\{2 N \log t+\left(\frac{4 N}{\alpha}-2\right) \log t\right\}^{1 / 2}
$$

and

$$
f_{2}(t)=\left\{2 N \log t+\left(\frac{4 N}{\alpha}+2\right) \log _{(2)} t\right\}^{1 / 2} .
$$

A computation shows that

$$
I\left(f_{1}\right)=\infty \quad \text { and } \quad I\left(f_{2}\right)<\infty .
$$

Then we have

Lemma 1. The theorem holds under the general situation if it is valid only for $f(t) \in F$, i.e. it suffices to prove it only for $f(t) \in F$.

Proof. For a function $\varphi(t)$ cited in theorem, set

$$
\hat{\varphi}(t)=\left(\varphi(t) \vee f_{1}(t)\right) \wedge f_{2}(t)^{*} .
$$

We see easily that $\hat{\varphi}(t) \in F$.

Case 1: $I(\varphi)<\infty$. For any monotone increasing sequence $\left\{t_{m}\right\}$ it holds for all sufficiently large $m$

$$
\varphi\left(t_{m}\right)>f_{1}\left(t_{m}\right)
$$

In fact, if there exists a monotone increasing sequence $\left\{\ddot{t}_{m}\right\}$ such that for any $m \quad \varphi\left(t_{m}\right) \leqq f_{1}\left(t_{m}\right)$, it yields a contradiction as follows. Set

Evaluate the integral

$$
\begin{aligned}
& t_{e}=\min \left\{t ; \varphi(t) \geqq\left(\frac{4 N}{\alpha}-1\right)^{1 / 2}\right\}, \\
& t_{n} \geqq\left(2 t_{e} \vee e^{e}\right) .
\end{aligned}
$$

$$
\int_{t_{e}}^{t_{n}} t^{N-1} \cdot K[\varphi(t)] d t
$$

*) $a \bigvee b=\max (a, b), a \wedge b=\min (a, b)$ 
which is not greater than $I(\varphi)$. Since $K[x]$ is monotone decreasing for $x \geqq\left(\frac{4 N}{\boldsymbol{\alpha}}-1\right)^{1 / 2}$, we have by the assumption that

$$
\begin{aligned}
I(\varphi) & \geqq \frac{1}{N}\left(t_{n}^{N}-t_{e}^{N}\right) \cdot K\left[f_{1}\left(t_{n}\right)\right] \\
& \geqq \frac{1}{N}(2 N)^{\frac{1}{2}\left(\frac{4 N}{\alpha}-1\right)} \cdot\left(1-\frac{1}{2^{N}}\right) \cdot\left(\log t_{n}\right)^{1 / 2} \rightarrow \infty, n \rightarrow \infty
\end{aligned}
$$

which contradicts the assumption, $I(\varphi)<\infty$. Hence, for large $t$, we obtain (2. 2), which implies

$$
\hat{\varphi}(t) \leqq \varphi(t)
$$

Moreover, we have

$$
I(\hat{\varphi})<\infty \text {. }
$$

Really, separating the domain of integration of $I(\hat{\varphi})$ into two parts; $\left\{t ; \varphi<f_{2}\right\}$ and $\left\{t ; \varphi \geqq f_{2}\right\}$, it holds

$$
I(\hat{\varphi}) \leqq I(\varphi)+I\left(f_{2}\right),
$$

where both in the right-hand side are finite. Therefore, from (2.3) we can conclude $\varphi(t) \in U^{u}$ if we can show that (2.4) implies $\hat{\varphi}(t) \in U^{u}$.

Case 2: $I(\varphi)=\infty$. In this case $I(\hat{\varphi})=\infty$. Actually, if there exists a monotone increasing sequence $\left\{t_{n}\right\}$ for which $\varphi\left(t_{n}\right)<f_{1}\left(t_{n}\right)$ holds for all sufficiently large $n$, the fact $I(\hat{\varphi}) \rightarrow \infty$ is similarly shown as well as in the Case 1. On the other hand, if $f_{1}(t)<\varphi(t)$ for large $t$, then we have $\hat{\varphi}(t) \leqq \varphi(t)$. Using the monotone property of the function $K[x]$ for $x \geqq\left(\frac{4 N}{\alpha}-1\right)^{1 / 2}$, we evaluate by Lemma 5 (a) below

$$
\begin{aligned}
I(\hat{\varphi}) & =\int_{e}^{\infty} t^{N-1} \cdot K[\hat{\varphi}(t)] d t \\
& \geqq \int_{e^{2 / \alpha}}^{\infty} t^{N-1} \cdot K[\varphi(t)] d t \\
& =I(\varphi)-\int_{e}^{e^{2 / \alpha}} t^{N-1} \cdot K[\varphi(t)] d t \\
& =\infty
\end{aligned}
$$

Thus we have $I(\hat{\varphi})=\infty$ in either case. In the sequel it suffices to prove 
that $\hat{\varphi}(t) \in L^{u}$ implies $\varphi(t) \in L^{u}$. The assumption $\hat{\varphi}(t) \in L^{u}$ implies that for almost all $w$ and every positive $\varepsilon_{1}$, a pair $(A, B)$ can be found such that $d(A, B)<\varepsilon_{1}$ and

$$
|x(A, w)-x(B, w)|>\sigma(A, B) \cdot \hat{\varphi}(1 / d(A, B))
$$

happens. On the other hand, since $I\left(f_{2}\right)<\infty$ and $f_{2}(t) \in F$, we know $f_{2}(t) \in U^{u}$ in view of the assumption of Lemma 1 . This means that for almost all $w$, there exists $\varepsilon_{2}(w)>0$ such that if $d(A, B)<\varepsilon_{2}(w)$, then

$$
|x(A, w)-x(B, w)| \leqq \sigma(A, B) \cdot f_{2}(1 / d(A, B)) .
$$

From (2.5) and (2.6) we obtain that for $d\left(A_{n}, B_{n}\right)<\varepsilon(w)$ for all large $n$ it holds

$$
\hat{\varphi}\left(1 / d\left(A_{n}, B_{n}\right)\right)<f_{2}\left(1 / d\left(A_{n}, B_{n}\right)\right) .
$$

Relying on the definition of $\hat{\varphi}(t)$ we have for $d(A, B) \leqq \varepsilon(w)$

$$
\hat{\varphi}\left(1 / d\left(A_{n}, B_{n}\right)\right) \geqq \varphi\left(1 / d\left(A_{n}, B_{n}\right)\right),
$$

which implies

$$
\varphi(t) \in L^{u} \text {. }
$$

3. We define the point-sets as follows, which are really the set of partition-point of $D$. Using these point-sets, the sequence of events will be defined. Several evaluations for upper and lower classes will be developed by these terms.

Let $p$ take integers $; p-\left[e^{3 / \alpha}\right]^{*)}=1,2,3, \cdots \cdots$. Set the point-sets for each $p$ :

$$
\begin{aligned}
& \boldsymbol{B}^{(p)}=\left\{B ; B=\left(k_{i} / 2^{p}\right) \in D, k_{i}= \pm 1, \cdots \pm 2^{p}, i=1,2, \cdots, N\right\} \\
& \boldsymbol{L}^{(p)}=\left\{L ; L=\left(l_{i} / 2^{p}\right), \quad l_{i}=0, \pm 1, \pm 2, \cdots, \quad i=1,2, \cdots, N\right\} \\
& \boldsymbol{A}^{(p)}=\left\{A ; A=B+L \in D, B \in B^{(p)}, L \in \boldsymbol{L}^{(p)}, 1 / 3 v_{p} \leqq d(A, B) \leqq 1 / v_{p}\right\}, \\
& \boldsymbol{Q}^{(p, d)}=\left\{Q ; Q=\left(m_{i}^{(d)} \cdot e^{-d c} / 2^{p}\right), m_{i}^{(d)}=0, \pm 1, \cdots, \pm e^{d c}, i=1,2, \cdots, N\right\} \text {, } \\
& d=1,2, \cdots,
\end{aligned}
$$

where $v_{p}=2^{p} / p^{1 / \alpha}$ and $c$ denotes some large number which makes $e^{c}$ an integer (c.f. Lemma 9 below) and $\left(a_{i}\right)$ denotes $\left(a_{1}, a_{2}, \cdots, a_{n}\right)$.

$$
\begin{aligned}
& \boldsymbol{A}^{(p, d)}=\left\{A^{\prime} ; A^{\prime}=A+Q \in D, A \in \boldsymbol{A}^{(p)}, Q \in \boldsymbol{Q}^{(p, d)}\right\}, \\
& \boldsymbol{B}^{(p, d)}=\left\{B^{\prime} ; B^{\prime}=B+Q \in D, \quad B \in \boldsymbol{B}^{(p)}, Q \in \boldsymbol{Q}^{(p, d)}\right\},
\end{aligned}
$$

*) $[x]$ indicates the integral part of $x$. 


$$
\begin{aligned}
& \boldsymbol{X}^{(p)}(A)=\left\{X ; X=\left(x_{i}\right), \quad\left(k_{i}+l_{i}-1\right) / 2^{p} \leqq x_{i} \leqq\left(k_{i}+l_{i}-1\right) / 2^{p},\right. \\
& \left.A=\left(k_{i}+l_{i} / 2^{p}\right) \in \boldsymbol{A}^{(p)}\right\}, \\
& \dot{\boldsymbol{Y}}^{(p)}(B)=\left\{Y ; Y=\left(y_{i}\right),\left(k_{i}-1\right) / 2^{p} \leqq y_{i} \leqq\left(k_{i}+1\right) / 2^{p}, \quad B=\left(k_{i} / 2^{p}\right) \in \boldsymbol{B}^{(p)}\right\} .
\end{aligned}
$$

For a function $\varphi(t)$, define the sequence of function $\left\{\lambda_{(t)}(\varphi)\right\}$ by

$$
\begin{aligned}
& \lambda_{(d)}(\varphi)(t)=\varphi(t)+\frac{2 N \cdot c}{\varphi(t)} \cdot \sum_{l=0}^{d-1}\left(1 / 2^{\alpha}\right)^{l}, d=1,2, \cdots, \\
& \lambda_{(\infty)}(\varphi)(t)=\varphi(t)+\frac{2 N \cdot c^{\prime}}{\varphi(t)}, \quad c^{\prime}=2^{\alpha} \cdot c /\left(2^{\alpha}-1\right) .
\end{aligned}
$$

Further, we define the three types of events by

(1) for $P \in \boldsymbol{A}^{(p)}$ and $Q \in \boldsymbol{B}^{(p)}$

$$
E(P, Q)=\{w ; x(P, w)-x(Q, w)>\sigma(P, Q) \cdot \varphi(1 / d(P, Q))\},
$$

(2) for $P=A+Q_{1} \in A^{(p, d)}$ and $Q=B+Q_{2} \in B^{(p, d)}$

$$
F^{(d)}(P, Q)=\left\{w ; x(P, w)-x(Q, w)>\sigma(P, Q) \cdot \lambda_{(d-1)}(\varphi)(1 / d(A, B))\right\},
$$

(3) for $A=B+L \in \boldsymbol{A}^{(p)}$ and $B \in \boldsymbol{B}^{(p)}$

$$
\widetilde{E}(A, B)=\left\{w \max _{\substack{P \in X^{(p)}(A) \\ Q \in Y^{(p)}(B)}}(x(P, w)-x(Q, w)) / \sigma(P, Q) \geqq \lambda_{(\infty)}(\varphi)(1 / d(A, B))\right\} .
$$

For the collection of events; $\mathscr{E}=\left\{E(P, Q) ; P \in A^{(p)}, Q \in B^{(p)}, P=Q+L\right.$, $\left.L \in \boldsymbol{L}^{(p)}\right\}$, we shall order as follows where $\mathscr{E}$ is defined: If $E_{n}=E(P, Q) \in \mathscr{E}$ where $P=\left(k_{i}+l_{i} / 2^{p}\right) \in \boldsymbol{A}^{(p)}, \quad Q=\left(k_{i} / 2^{p}\right) \in \boldsymbol{B}^{(p)}$ and if $E_{m}=E\left(P^{\prime}, Q^{\prime},\right) \in \mathscr{E}$ where $P^{\prime}=\left(k_{i}^{\prime}+l_{i}^{\prime} / 2^{p}\right) \in \boldsymbol{A}^{\left(p^{\prime}\right)}, Q^{\prime}=\left(k_{i}^{\prime} / 2^{p}\right) \in \boldsymbol{B}^{\left(p^{\prime}\right)}$, then $n<m$ holds if and only if
(a) $\quad p<p^{\prime}$

or

(b) $\quad\left\|l^{\prime}\right\|<\|l\|$ when $p=p^{\prime}$

or

(c) $\quad k_{i}<k_{i}^{\prime}(i \leqq N)$ when $p=p^{\prime},\left\|l^{\prime}\right\|=\|l\|$ and $k_{j}=k_{j}^{\prime}(j=1, \cdots, i-1)$

or

(d) $\quad l_{i}<l_{i}^{\prime}(i \leqq N)$ when $p=p^{\prime},\left\|l^{\prime}\right\|=\|l\|, k_{i}=k_{i}^{\prime}$ for all $i$ and $l_{j}=l_{j}^{\prime}(j=1,2, \cdots, i-1)$, 
where $\|l\|$ denotes $\left(\sum_{i=1}^{N} l_{i}^{2}\right)^{1 / 2}$.

Also we give the same numbering for event in $\tilde{\mathscr{E}}=\left\{\tilde{E}(A, B) ; A \in \boldsymbol{A}^{(p)}\right.$, $B \in \boldsymbol{B}^{(p)}, A=B+L$, for all $\left.p\right\}$, i.e. when $E_{n}=E(A, B)$, then $\widetilde{E}_{n}=\tilde{E}(A, B)$.

Under these preparations Lemma 2 can be stated as follows;

\section{LemMA 2.}

[2. 1] If $I(\varphi)<\infty$, then $\sum_{n=1}^{\infty} P\left(\tilde{E}_{n}\right)<\infty$.

[2. 2] If $I(\varphi)=\infty$, then $\sum_{n=1}^{\infty} P\left(E_{n}\right)=\infty$.

[2. 3] For each $n, \lim _{m \rightarrow \infty} \rho\left(u_{n}, u_{m}\right)=0$,

where

$$
u_{k}=\left(x\left(A_{k}\right)-x\left(B_{k}\right)\right) / \sigma\left(A_{k}, B_{k}\right)
$$

for $A_{k}=P, B_{k}=Q$, respectively if $E_{k}=E(P, Q) \in \mathscr{E}$, and $\rho\left(u_{n}, u_{m}\right)$ denotes the correlation-coefficient between $u_{n}$ and $u_{m}$.

[2. 4] There exist two absolute constants $k_{1}$ and $k_{2}$ with the following property: to each $E_{j}$ there corresponds a finite set of events $\boldsymbol{E}_{j}=\left\{E_{j 1}, E_{j 2}, \cdots, E_{j s(j)},\right\}\left(E_{j i} \in \mathscr{E}\right)$ such that

$$
\sum_{i=1}^{s(j)} P\left(E_{j} \cap E_{j i}\right)<k_{1} \cdot P\left(E_{j}\right)
$$

and if $E_{k}$ is not in $E_{j}(k>j)$, then

$$
P\left(E_{j} \cap E_{k}\right)<k_{2} \cdot P\left(E_{j}\right) \cdot P\left(E_{k}\right) .
$$

4. (Proof of theorem). In view of Lemma 1 and symmetric property of the process $\{x(A, w) ; A \in D\}$ it is enough to prove only for $\varphi(t) \in F$ and for the events defined in $\mathbf{3}$.

Case 1: $I(\varphi)<\infty$. From Lemma 2, [2,1] and by Borel-Cantelli lemma, for almost all $w$ there exists the number $n_{0}(w)$, namely $p_{0}(w)$ such that for any $p>p_{0}(w), \tilde{E}(A, B)\left(A \in \boldsymbol{A}^{(p)}, B \in \boldsymbol{B}^{(p)}\right)$ can not occur. Set

$$
p_{1}(w)=p_{0}(w) \vee([6 \sqrt{N}]+1) \vee\left(\left[\frac{10 c^{\prime}}{\alpha \log 2}+2 \sqrt{N}\right]+1\right) .
$$

Take any points $A$ and $B$ satisfying the relation

$$
d(A, B)<\left(p_{1}^{1 / \alpha}(w)-2 \sqrt{N}\right) / 2^{p_{1}(w)} .
$$


Then, since $\left(x^{1 / \alpha}-2 \sqrt{N}\right) / 2^{x}$ is monotone decreasing for $x>\frac{1}{\alpha \log 2}+2 \sqrt{N}$, there exists a integer $p\left(\geqq p_{1}\right)$ such that

$$
\left((p+1)^{1 / \alpha}-2 \sqrt{N}\right) / 2^{p+1} \leqq d(A, B) \leqq\left(p^{1 / \alpha}-2 \sqrt{N}\right) / 2^{p}
$$

holds. For this $p$, choose points $A^{\prime} \in \boldsymbol{A}^{(p)}$ and $B^{\prime} \in \boldsymbol{B}^{(p)}$ satisfying the following both conditions;

$$
\begin{aligned}
d(A, B) & \leqq d\left(A^{\prime}, B^{\prime}\right), \\
\left\{d\left(A, A^{\prime}\right)+d\left(B, B^{\prime}\right)\right\} & =\min _{\substack{X \in A^{(p)} \\
Y \in B^{(p)}}}\{d(A, X)+d(B, Y)\} .
\end{aligned}
$$

Then it holds clearly that

$$
d\left(A, A^{\prime}\right) \leqq \sqrt{N} / 2^{p} \quad \text { and } \quad d\left(B, B^{\prime}\right) \leqq \sqrt{N} / 2^{p} .
$$

Hence by (4.2) we have

$$
1 / 3 v_{p} \leqq d\left(A^{\prime}, B^{\prime}\right) \leqq 1 / v_{p} .
$$

Since this implies $E\left(A^{\prime}, B^{\prime}\right) \in \mathscr{E}$, we can see that the event $\tilde{E}\left(A^{\prime}, B^{\prime}\right)$ belongs to $\tilde{\mathscr{E}}$. We have, therefore, because of (4.1) and (4.3)

$$
x(A, W)-x(B, W) \leqq \sigma(A, B) \cdot \lambda_{(\infty)}(\varphi)\left(1 / d\left(A^{\prime}, B^{\prime}\right)\right) .
$$

Since $\lambda_{(\infty)}(\varphi)\left(v_{p}\right)$ is monotone decreasing for $p>10 c^{\prime} / \log 2$, we obtain by (4. 3)

$$
x(A, W)-x(B, W) \leqq \sigma(A, B) \lambda_{(\infty)}(\varphi)(1 / d(A, B)),
$$

which for (4. 1) implies

$$
\varphi(t)+\frac{2 N \cdot c^{\prime}}{\varphi(t)} \in U^{u}
$$

In order to assure that $\varphi(t)$ itself belongs to $U^{u}$, we set

$$
\eta(t)=\varphi(t)-\frac{10 N c^{\prime}}{\varphi(t)} \text {. }
$$

Then a computation show that $I(\eta) \leqq e^{10 N c^{\prime}} \cdot I(\varphi)$. Accordingly $I(\eta)<\infty$ holds under the condition $I(\varphi)<\infty$.

This implies from the above argument that

$$
\eta(t)+\frac{2 N c^{\prime}}{\eta(t)} \in U^{u}
$$


Since for large $t$

$$
\eta(t)+\frac{2 N c^{\prime}}{\eta(t)} \leqq \varphi(t),
$$

we have $\varphi(t) \in U^{u}$. This completes the proof of theorem in the case $I(\varphi)<\infty$.

Case 2: $I(\varphi)=\infty$. First of all we shall show that for elements of $\mathscr{E} ; E_{h}, E_{h+1}, \cdots, E_{h+(n-h)}(m \geqq n \geqq h)$ it holds for large $m$,

$$
P\left(E_{m} / E_{h}^{\prime} \cap \cdots \cap E_{h+(n-h)}^{\prime}\right)>\frac{1}{8} \cdot P\left(E_{m}\right),
$$

where $E_{k}^{\prime}$ denotes the complement of $E_{k}$ and $P\left(E_{m} / C\right)$ the conditional probability of $E_{m}$ under the condition $C$. Corresponding to $E_{m}(=E(A, B))$ and $E_{l}(=E(P, Q))(l=h, \cdots, n)$, we define their subsets $E_{m}^{*}$ and $\dot{E}_{l}$ by

$$
E_{m}^{*}=\{w ; \sigma(A, B) \cdot \varphi(1 / d(A, B))<x(A, w)-x(B, w)<2 \sigma(A, B) \cdot \varphi(1 / d(A, B))\},
$$

and

$$
\dot{E}_{l}=\left\{w ; x(P, w)-x(Q, w) \in B_{l}\right\}, l=h, \cdots, n,
$$

where $B_{l}$ is any bounded Borel set. If we set $\varepsilon(m)$ by

$$
P\left(E_{m}^{*} / \dot{E}_{h} \cap \cdots \cap \dot{E}_{n}\right)=(1+\varepsilon(m)) \cdot P\left(E_{m}^{*}\right),
$$

then $\varepsilon(m)$ is a function of $\rho\left(u_{l}, u_{m}\right),(l=h, \cdots, n)$ and of $\sigma(A, B) \cdot \varphi(1 / d(A, B))$. Then we obtain from Lemma 2, $[2,3]$

$$
\varepsilon(m) \longrightarrow 0 \quad \text { as } \quad \max _{l} \rho\left(u_{l}, u_{m}\right) \longrightarrow 0
$$

(T. Sirao [11]). Therefore, we have for large $m$,

$$
P\left(E_{m}^{*} / \dot{E}_{h} \cap \cdots \cap \dot{E}_{n}\right)>\frac{1}{2} \cdot P\left(E_{m}^{*}\right)
$$

On the otherhand, we see easily

$$
P\left(E_{m}\right)<2 P\left(E_{m}^{*}\right)
$$

for all large $m$. Further, if we denote the event that $u_{\imath}+a$ is positive by $E_{l}(a)$, it is clear that

$$
P\left(E_{l}(a)\right) \longrightarrow 1 \quad \text { as } \quad a \longrightarrow \infty \text {. }
$$


Choosing, therefore, $a_{h, n}$ for each pair of positive integers $h$ and $n(h \leqq n)$ such that

$$
P\left\{\bigcap_{l=h}^{n}\left(E_{l}^{\prime} \cap E_{l}\left(a_{h, n}\right)\right)\right\} \geqq P\left(\bigcap_{l=h}^{n} E_{l}^{\prime}\right) / 2,
$$

we have

$$
P\left(E_{m} / E_{h}^{\prime} \cap \cdots \cap E_{n}^{\prime}\right) \geqq P\left(E_{m} / \bigcap_{l=h}^{n}\left(E_{l}^{\prime} \cap E_{l}\left(a_{h, n}\right)\right) / 2 .\right.
$$

Therefore taking $\dot{E}_{l}=E_{l}^{\prime} \cap E_{l}\left(a_{h, n}\right),(l=h, \cdots, n)$ in (4.5), it yields (4. 4) (T. Sirao [11]). (4.4) together with Lemma 2, [2. 2] and Lemma 2, [2.4] implies that

$$
P\left(E_{n} \text { occur infinitely often }\right)=1,
$$

(K.L.Chung and P.Erdös [2]), which implies $\varphi(t) \in L^{u}$. This establishes the proof of theorem in the Case $2, I(\varphi)=\infty$.

5. (Proof of Lemma 2.) This section is devoted to prove Lemma 2. In the first place Lemma 2, [2. 1] is verified after several preparations. In the second place Lemma 2, [2. 2], [2.3] and [2.4] will be proved, which were required to refer K.L.Chung-P.Erdös lemma for the proof of lower class.

Following Lemma 3 will be cited so often from now on, but we shall omit the proof, because it is easy.

\section{Lemma 3.}

(a) For $x \geqq 1$,

$$
\frac{1}{2 x} \exp \left(-x^{2} / 2\right) \leqq \int_{x}^{\infty} \exp \left(-t^{2} / 2\right) d t \leqq \frac{1}{x} \exp \left(-x^{2} / 2\right) .
$$

(b) At $x=e, \log x / x$ attains its maximum and for $x>e$ it is monotone decreasing.

(c) $\log _{(2)} x / \log x$ attains its maximum at $x=e^{e}$ and monotone decreasing for $x>e^{e}$.

(d) For sufficiently large $x$ (e.g. $x \geqq e^{3 / \alpha}$ ) and for $0<\alpha \leqq 1$,

$$
(\alpha \cdot \log 2) \cdot(\log x / x)<1
$$

Lemma 4. For $\varphi(t) \in F$, it yields;

(a) For $t>e$, 


$$
(2 N \log t)^{1 / 2} \leqq \varphi(t) \leqq c_{1} \cdot(2 N \log t)^{1 / 2},
$$

where

$$
c_{1}=\left\{1+\left(\frac{4}{\alpha}+\frac{2}{N}\right) \frac{1}{2 e}\right\}^{1 / 2} .
$$

(b) For sufficiently large $p\left(e . g . p>e^{3 / \alpha}\right)$,

$$
\varphi\left(v_{p}\right)>1 \quad \text { and } \quad \varphi\left(3 v_{p}\right)>1 .
$$

(c) For the same $p$ in (b),

where

$$
\begin{aligned}
& c_{2} \cdot p \leqq \varphi^{2}\left(v_{p}\right) \leqq p \cdot\left\{c_{1}^{2} 2 N \log 2\right\}, \\
& c_{2} \cdot p \leqq \varphi^{2}\left(3 v_{p}\right) \leqq c_{3} \cdot p
\end{aligned}
$$

$$
c_{2}=2 N \cdot \log 2 \cdot\left\{1-\frac{3}{\alpha^{2} \cdot \log 2 \cdot e^{3 / \alpha}}\right\},
$$

and

$$
c_{3}=c_{1}^{2} \cdot 2 N \log 2 \cdot\left(1+\frac{\log 3}{2 \log 2}\right) \text {. }
$$

Proof. (a). Since $\varphi(t) \in F$ implies that

$$
\begin{aligned}
& (2 N \log t)^{1 / 2} \cdot\left\{1+\left(\frac{4}{\alpha}-\frac{2}{N}\right) \cdot \frac{\log _{(2)} t}{2 \log t}\right\}^{1 / 2} \leqq \varphi(t \\
& \leqq(2 N \cdot \log t)^{1 / 2} \cdot\left\{1+\left(\frac{4}{\alpha}+\frac{2}{N}\right) \cdot \frac{\log (2) t}{2 \log t}\right\}^{1 / 2},
\end{aligned}
$$

we obtain (a) due to Lemma 3, (c).

(b). From Lemma 4, (a) it is sufficient to choose so large $p$ that

$$
\begin{aligned}
& v_{p}>e, \text { i.e. } \\
& p \log 2-\frac{1}{\alpha} \log p>1 .
\end{aligned}
$$

Since from Lemma 3, (b) we have

$$
p \log 2\left\{1-\frac{\log p}{p} \cdot \frac{1}{\alpha \log 2}\right\}>p \cdot \log 2\left\{1-\frac{3}{\alpha^{2} \cdot \log 2 \cdot e^{3 / \alpha}}\right\}
$$

for $p>e^{3 / \alpha}>e$, we obtains (b). The latter of (b) could be checked similarly.

(c). From the above results (a) and (b), we have for $p>e^{3 / \alpha}$,

$$
2 N \cdot \log v_{p} \leqq \varphi^{2}\left(v_{p}\right) \leqq c_{1}^{2} 2 N \log v_{p} .
$$

If we set $c_{2}$ as cited above, we find (c). The latter part of (c) is similarly derived. 
Lemma 5. For $\varphi(t) \in F$ we have;

(a) For sufficiently large $t$ (e.g. $\left.t \geqq e^{\left.2\right|^{\alpha}}\right), K[\varphi(t)]$ is monotone decreasing in $t$.

(b) For $p>2 \cdot e^{3 / \alpha} / \alpha, K\left[\varphi\left(v_{p}\right)\right]$ is monotone decreasing in $p$ and so does $K\left[\varphi\left(3 v_{p}\right)\right]$.

Proof. (a). Since $K[x]$ is monotone decreasing for $x \geqq\left(\frac{4 N}{\alpha}-1\right)^{1 / 2}$, it suffices to find $t$ such that $\varphi(t) \geqq\left(\frac{4 N}{\alpha}-1\right)^{1 / 2}$.

By Lemma 4, (a) we can find it as follows;

$$
2 N \log t \geqq \frac{4 \dot{N}}{\alpha} \geqq \frac{4 N}{\alpha}-1, \quad \text { i.e. } \quad t \geqq e^{2 / \alpha}
$$

(b). In view of the above (a), it is enough to choose $p$ such that

$$
\begin{gathered}
1 / v_{p} \geqq e^{2 / \alpha}, \quad \text { i.e. } \\
p \cdot \log 2 \cdot\left\{1-\frac{\log p}{p} \cdot \frac{1}{\alpha \log 2}\right\} \geqq 2 / \alpha .
\end{gathered}
$$

Using the same argument as well as in the proof of Lemma 4, (b), we have (b) for $p \geqq 2 \cdot e^{3 / \alpha} / \alpha$. The remaining part is obtained similarly.

Lemma 6. For $E_{n}=E(A, B) \in \mathscr{E}$, we have

$$
\begin{array}{r}
\exp \left\{-\frac{1}{2} \varphi^{2}(1 / d(A, B))\right\} / 2 \sqrt{2 \pi} \cdot \varphi(1 / d(A, B)) \leqq p\left(E_{n}\right) \leqq \\
\leqq \\
\exp \left\{-\frac{1}{2} \varphi^{2}(1 / d(A, B))\right\} / \sqrt{2 \pi} \cdot \varphi(1 / d(A, B)) .
\end{array}
$$

Proof. It is evident from Lemma 4, (b), Lemma 3, (a) and from the fact;

$$
\sqrt{2 \pi} \cdot P\left(E_{n}\right)=\int_{\varphi(1 / d(A, B))}^{\infty} \exp \left(-t^{2} / 2\right) d t .
$$

Lemma 7. If $I(\varphi)<\infty$, then we have

$$
\sum_{n=1}^{\infty} P\left(E_{n}\right)<\infty
$$

Proof. Set the number corresponding to $p_{0}=\left[2 e^{3 / \alpha} / \alpha\right]+1$ by $n_{0}$. It is enough to check only the following; 


$$
\begin{gathered}
\sum_{n=n_{0}}^{\infty} P\left(E_{n}\right) \leqq \sum_{\substack{E(A, B) \in \in \mathbb{E} \\
\text { for } p=p_{0}}} P(E(A, B)) \\
\leqq \frac{1}{\sqrt{2 \pi}} \cdot \sum_{p=p_{0}}^{\infty}\left(2.2^{p}\right)^{N} \cdot\left(p^{1 / \alpha}\right)^{N} \cdot \exp \left\{-\frac{1}{2} \varphi^{2}(1 / d(A, B)\} / \varphi(1 / d(A, B)) .\right.
\end{gathered}
$$

By Lemma 5, (b) and Lemma 4, (c)

$$
\begin{aligned}
& \sum_{n=n_{0}}^{\infty} P\left(E_{n}\right) \leqq c_{4} \sum_{p=p_{0}}^{\infty} v_{p}^{N-1} \cdot\left(v_{p}-v_{p-1}\right) p^{2 N / \alpha} \exp \left(-\frac{1}{2} \varphi^{2}\left(v_{p}\right)\right) / \varphi\left(v_{p}\right) \\
& \leqq c_{4} \cdot c_{2}^{-2 N l_{\alpha}} \cdot \sum_{p=p_{0}}^{\infty} \int_{v_{p-1}}^{v_{p}} v_{p}^{N-1} K\left[\varphi\left(v_{p}\right)\right] d t \\
& \leqq 2^{N-1} \cdot c_{4} \cdot c_{2}^{-2 N / x} \sum_{p=p_{0}}^{\infty} \int_{v_{p-1}}^{v_{p}} t^{N-1} K[\varphi(t)] d t
\end{aligned}
$$

As a consequence, we obtain

$$
\begin{aligned}
P\left(E_{n}\right) & \leqq 2^{N-1} c_{4} \cdot c_{2}^{-2 N / \alpha} \cdot \int_{v_{p_{0}-1}}^{\infty} t^{N-1} K[\varphi(t)] d t \\
& \leqq 2^{N-1} \cdot c_{4} \cdot c_{2}^{-2 N / \alpha} \cdot I(\varphi),
\end{aligned}
$$

which completes the proof of Lemma 7.

The above Lemma 7 plays an essential role to prove Lemma 2, [2. 1].

Lemma 8. For any pair of points $(A, B)$, where $A \in A^{(p, d)}, B \in B^{(p, d)}$ and $A-B=L+Q_{1}+Q_{2}, L \in L^{(p)}, Q_{1}, Q_{2} \in Q^{(p, d)}$, if we choose properly a pair $\left(A^{\prime}, B^{\prime}\right)$ such that $A^{\prime} \in A^{(p, d-1)}$ and $B^{\prime} \in B^{(p, d-1)}$, and $A^{\prime}-B^{\prime}=L+Q_{1}^{\prime}+Q_{2}^{\prime}$ $\left(Q_{1}^{\prime}\right.$ and $\left.Q_{2}^{\prime} \in Q^{(p, d-1)}\right)$, we have

$$
\rho\left(u, u^{\prime}\right) \geqq 1-(1+\alpha)\left\{3 \sqrt{N} / \sqrt{1-\frac{12 \sqrt{N}}{p^{1 / \alpha}}} \cdot p^{1 / \alpha} e^{(d-1) c}\right\}^{\alpha},
$$

where $u=x(A)-x(B), u^{\prime}=x\left(A^{\prime}\right)-x\left(B^{\prime}\right)$ and $p \geqq\left[12 \sqrt{N} e^{3 / \alpha}\right]+1$.

Proof. Choose $A^{\prime}$ and $B^{\prime}$ as follows: If $i$-th coordinate of point $A$ is not smaller than $i$-th coordinate of point $B$, that is, if $k_{i}+l_{i}+m_{i}^{(d)} \dot{e}^{-d c} \geqq$ $k_{i}+n_{i}^{(d)} e^{-d c}$, then we choose the point $A^{\prime} \in \boldsymbol{A}^{(p, d-1)}$ whose $i$-th coordinate is greater than i-th coordinate of $A$ and has the minimum distance, i.e. 


$$
A^{\prime}=\left(\left(k_{i}+l_{i}+m_{i o}^{(d-1)} \cdot e^{-(d-1) c}\right) / 2^{p}\right) \text {, for which }
$$

$$
\begin{gathered}
m_{i o}^{(d-1)} e^{-(d-1) c}>m_{i}^{(d)} e^{-d c}, \\
m_{i o}^{(d-1)} \cdot e^{-(d-1) c}-m_{i}^{(d)} e^{-d c} \leqq m_{i}^{(d-1)} \cdot e^{-(d-1) c}-m_{i}^{(d)} e^{-d c}
\end{gathered}
$$

for any $A=\left(\left(k_{i}+l_{i}+m_{i}^{(d-1)} \cdot e^{-(d-1) c}\right) / 2^{p}\right) \in A^{(p, d-1)}$.

Further choose the point $B^{\prime} \in \boldsymbol{B}^{(p, d-1)}$, whose i-th coordinate is smaller than i-th coordinate of $B$ and has the minimum distance, i.e.

$$
B^{\prime}=\left\{\left(k_{i}+n_{i o}^{(d-1)} \cdot e^{-(d-1) c}\right) / 2^{p}\right\}
$$

for which

$$
n_{i o}^{(d-1)} \cdot e^{-(d-1) c}<n_{i}^{(d)} \cdot e^{-d c},
$$

for any $B=\left\{\left(k_{i}+n_{i}^{(d-1)} \cdot e^{-(d-1) c}\right) / 2^{p}\right\} \in B^{(p, d-1)}$.

In the other case, i.e. if $\mathrm{i}$-th coordinate of $A$ is smaller than that of $B$, then choose the points $A^{\prime}$ and $B^{\prime}$ whose coordinates satisfy the reversed inequality respected to (5.2) and (5.3), respectively. For points chosen as above, we see

$$
d\left(A, A^{\prime}\right) \leqq \sqrt{N} e^{-(d-1) c} / 2^{p}, d\left(B, B^{\prime}\right) \leqq \sqrt{N} e^{-(d-1) c} / 2^{p},
$$

(5. 4)

$$
d(A, B) \leqq d\left(A^{\prime}, B\right) \quad \text { and } \quad d(A, B) \leqq d\left(A, B^{\prime}\right) .
$$

Then we have

$$
\begin{aligned}
& \quad \begin{aligned}
\rho\left(u, u^{\prime}\right) & \geqq \frac{\left\{2 \cdot d^{\alpha}(A, B)-d^{\alpha}\left(A, A^{\prime}\right)-d^{\alpha}\left(B, B^{\prime}\right)\right\}}{2\left\{d^{\alpha}(A, B) \cdot d^{\alpha}\left(A^{\prime} B^{\prime}\right)\right\}^{1 / 2}} \\
& \geqq 1-(1+\alpha) m^{\alpha}, \\
\text { where } \quad m & =d\left(A, A^{\prime}\right)\left|d(A, B) \vee d\left(B, B^{\prime}\right)\right| d(A, B) .
\end{aligned}
\end{aligned}
$$

In order to estimate $m$, it requires to evaluate $d(A, B)$ :

$$
\begin{aligned}
\left(2^{p}\right)^{2} \cdot d(A, B)^{2} & =\sum_{i=1}^{N}\left\{l_{i}+\left(m_{i}^{(d)}-n_{i}^{(d)}\right) e^{-d c}\right\}^{2} \\
& \geqq\left(\sum_{i=1}^{N} l_{i}^{2}\right) \cdot\left\{1-\frac{2 \cdot \sum_{i=1}^{N} l_{i}\left(m_{i}^{(d)}-n_{i}^{(d)}\right) e^{-d c}}{\left(\sum_{i=1}^{N} l_{i}^{2}\right)}\right\}
\end{aligned}
$$




$$
\begin{aligned}
& \geqq\left(\sum_{i=1}^{N} l_{i}^{2}\right) \cdot\left\{1-\frac{2\left\{\sum_{i=1}^{N}\left(m_{i}^{(d)}-n_{i}^{(d)}\right)^{2} e^{-2 d c}\right\}^{1 / 2}}{\left(\sum_{i=1}^{N} l_{i}^{2}\right)^{1 / 2}}\right\} \\
& \geqq\left(\sum_{i=1}^{N} l_{i}^{2}\right) \cdot\left\{1-\frac{4 \sqrt{N}}{\left(\sum_{i=1}^{N} l_{i}^{2}\right)^{1 / 2}}\right\} \\
& \geqq\left(\frac{1}{3} p^{1 / \alpha}\right)^{2}\left(1-\frac{4 \sqrt{N}}{\frac{1}{3} p^{1 / \alpha}}\right) .
\end{aligned}
$$

From this and (5. 4) we have Lemma 8.

In the following Lemma 9 , notation $\boldsymbol{A}^{(p, d)}$ and $\boldsymbol{B}^{(p, d)}$ are used for the fixed points $A$ and $B$ in their definition, respectively.

Lemma 9. For some fixed points $A$ and $B$

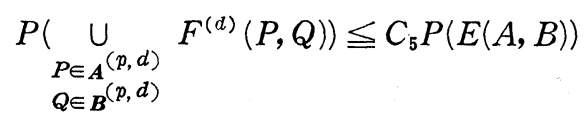

where $p=\left[12 N e^{3 / \alpha}\right]+1$ and $c_{5}$ is an absolute constant.

Proof. We shall prove by induction on $d$. By de Morgan's law, we see

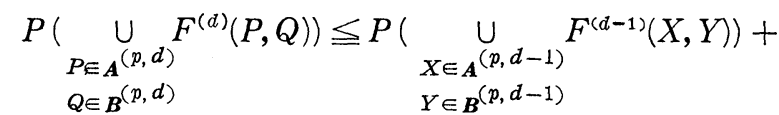

$$
\begin{aligned}
& +\sum_{\substack{P \in \boldsymbol{A}^{(p, d)} \\
Q \in B^{(p, d)}}} P\left(\bigcap_{\substack{X \in \boldsymbol{A}^{(p, d-1)} \\
Y \in \boldsymbol{B}^{(p, d-1)}}} F^{\prime(d-1)}(X, Y) \cap F^{(d)}(P, Q)\right) .
\end{aligned}
$$

Set $s=1 / d(A, B)$. For the case $d=1$ we can estimate as follows: For $P \in \boldsymbol{A}^{(p, 1)}$ and $Q \in \boldsymbol{B}^{(p, 1)}$, we have by Lemma 4, (b) and Lemma 3

$$
\begin{aligned}
P\left(F^{(1)}(P, Q)\right) & \leqq \frac{1}{\sqrt{2 \pi}}\left(\varphi(s)+\frac{2 N c}{\varphi(s)}\right)^{-1} \exp \left\{-\frac{1}{2}\left(\varphi(s)+\frac{2 N c}{\varphi(s)}\right)^{2}\right\} \\
& \leqq \frac{e^{-2 N c}}{\sqrt{2 \pi}} \cdot \frac{1}{\varphi(s)} \exp \left\{-\frac{1}{2} \varphi^{2}(s)\right\} \\
& \leqq 2 e^{-2 N c} P(E(A, B)) .
\end{aligned}
$$

Since the number of all combinations of $P \in \boldsymbol{A}^{(p, 1)}$ and $Q \in \boldsymbol{B}^{(p, 1)}$ does not exceed $\left(2 e^{c}\right)^{N} \times\left(2 e^{c}\right)^{N}$, we obtains 


$$
P\left(\underset{\substack{P \in \boldsymbol{A}^{(p, 1)} \\ Q \in \boldsymbol{B}^{(p, 1)}}}{\cup} F^{(1)}(P, Q)\right) \leqq 2 \cdot 4^{N} P(E(A, B) .)
$$

Next we consider the second term in (5.5). If we choose the pair $\left(P_{0}, Q_{0}\right), P_{0} \in \boldsymbol{A}^{(p, d-1)}, Q_{0} \in \boldsymbol{B}^{(p, d-1)}$ as in Lemma 8 corresponding to each pair $(P, Q), P \in \boldsymbol{A}^{(p, d)}, Q \in \boldsymbol{B}^{(p, d)}$, which satisfy (5.1), then we can see that

$$
\bigcap_{\substack{X \in A^{(p, d-1)} \\ Y \in B^{(p, d-1)}}}\left\{F^{(d-1)}(X, Y) \cap F^{(d)}(P, Q)\right\} \subset F^{(d-1)}\left(P_{0}, Q_{0}\right) \cap F^{(d)}(P, Q)
$$

since the set $\left\{(X, Y) ; X \in \boldsymbol{A}^{(p, d-1)}, Y \in \boldsymbol{B}^{(p, d-1)}\right\}$ contains $\left(P_{0}, Q_{0}\right)$. Hence in order to evaluate second term in (5.5), it suffices to evaluate the probability of the right-hand side above:

$$
P\left(F^{\prime(d-1)}\left(P_{0}, Q_{0}\right) \cap F^{(d)}(P, Q)\right)=\frac{1}{\sqrt{2 \pi}} \int_{\lambda_{(d-1)}}^{\infty} P\left[W \leqq \frac{\lambda(d-2)-\rho t^{2}}{\sqrt{1-\rho^{2}}}\right] \cdot e^{-\frac{t^{2}}{2}} d t,
$$

where we write $\lambda_{(k)}$ briefly instead of $\lambda_{k}(\varphi)(1 / d(A, B))$ for fixed $A$ and $B$, and $W$ (and $V$ below) are mutually independent random variables of standard normal distribution. Since the last integral is monotone decreasing in $\rho$ (T. Sirao [11]) and by replacing $\rho$ by $\rho_{0}$ which is set by the right-hand side in (5.1), we find

$$
\begin{gathered}
P\left(F^{\prime(d-1)}\left(P_{0}, Q_{0}\right) \cap F^{(d)}(P, Q)\right) \leqq P(V>\varphi(s)) \cdot P\left(W \geqq-\left(1-\rho_{0}^{2}\right)^{1 / 2} \cdot \lambda(d-2)+\right. \\
\left.+\left\{\left(1-\rho_{0}^{2}\right)^{-1 / 2} \cdot \rho_{0} \cdot 2 N c / \varphi(s) 2^{\alpha(d-1)}\right\}\right)
\end{gathered}
$$

where $\quad s=1 / d(A, B)$.

Further, for $p \geqq 12 \sqrt{N} \cdot e^{3 / \alpha}$ we estimate using (5.1)

$$
\begin{aligned}
& \left(1-\rho_{0}^{2}\right)^{1 / 2} \cdot \lambda(d-2) \\
& \leqq\left(6 \sqrt{N(1+\alpha))^{1 / 2}} \cdot\left\{\left(1-\frac{12 \sqrt{N}}{p^{1 / \alpha}}\right)^{1 / 4} \sqrt{p} \cdot e^{(d-1) c / 2}\right\}^{-1} \cdot\left\{\varphi(s)+\frac{2 N c}{\varphi(s)} \cdot \sum_{l=0}^{d-2}\left(\frac{1}{2^{\alpha}}\right)^{l}\right\}\right. \\
& \leqq(6 \sqrt{N}(1+\alpha))^{1 / 2} \cdot\left\{\left(1-\frac{12 \sqrt{N}}{p^{1 / \alpha}}\right)^{1 / 4} \cdot \sqrt{p} \cdot e^{(d-1) c / 2}\right\}^{-1} \cdot\left\{\sqrt{p} \cdot c_{1} \cdot(2 N \log 2)^{1 / 2}+\frac{2 N c^{\prime}}{\sqrt{c_{2} p}}\right\} \\
& \leqq(6 N(1+\alpha))^{1 / 2} \cdot\left\{c_{1}(2 N \log 2)^{1 / 2}+\frac{2 N c^{\prime}}{\sqrt{c_{2}} \cdot e^{3 / \alpha}}\right\} /\left(1-\frac{1}{e^{3 / \alpha}}\right)^{1 / 4} \equiv c_{6} \quad \text { (say) }
\end{aligned}
$$

In view of (5.1) and Lemma 4, (c) we can estimate similarly; 


$$
2 N c\left(1-\rho_{0}\right)^{-1 / 2} \cdot \rho_{0} / 2^{\alpha(d-1)} \cdot \varphi(s) \geqq c_{7} \cdot\left(e^{c / 2} / 2\right)^{\alpha(d-1)},
$$

where

$$
c_{7}=\frac{2 N c}{(6 \sqrt{N}(1+\alpha))^{1 / 2} \cdot c_{1} \cdot(2 N \log 2)^{1 / 2}} \cdot\left\{1-\frac{(1+\alpha)}{\sqrt{1-\frac{1}{e^{3 / \alpha}} \cdot e^{3 / \alpha}}}\right\}\left(1-\frac{1}{e^{3 / \alpha}}\right)^{1 / 4} .
$$

As a consequence we have

$$
P\left(F^{\prime(d-1)}\left(P_{0}, Q_{0}\right) \cap F^{(d)}(P, Q)\right) \leqq P(V \geqq \varphi(s)) \cdot P\left(W \geqq-c_{6}+c_{7}\left(e^{c / 2} / 2\right)^{\alpha(d-1)}\right) .
$$

In order to apply Lemma 3, (a) to the above, it is required to set $c$ such that

$$
-c_{6}+c_{7}\left(e^{c / 2} / 2\right)^{\alpha(d-1)}>1 .
$$

But for this it is enough to take $c$ such that

$$
c>\left\{\frac{2}{\alpha} \cdot \log \left(1+\frac{c_{6}^{\prime}}{c_{7}^{\prime}}\right)+\log 2\right\}+1
$$

where $c_{6}^{\prime}$ and $c_{7}^{\prime}$ are constants dependent on $c_{6}$ and $c_{7}$, respectively. Choose such $c$ and set $c_{8}$ by $c_{8}=c_{7}-\frac{c_{6}}{\left(e^{c / 2} / 2\right)^{\alpha(d-1)}}$.

Then we obtain

$$
\begin{gathered}
P\left(F^{\prime(d-1)}\left(P_{0}, Q_{0}\right) \cap F^{(d)}(P, Q)\right) \\
\leqq P(E(A, B)) \cdot\left(2 / e^{c / 2}\right)^{\alpha(d-1)} \cdot \exp \left(-\frac{c_{8}^{2}}{2}\left(\frac{e^{c / 2}}{2}\right)^{2 \alpha(d-1)}\right) / c_{8} \\
\leqq c_{9} e^{-4 d N c} \cdot P(E(A, B)),
\end{gathered}
$$

where $\quad c_{9}=2^{l_{0}} \cdot l_{0} ! / c_{8}^{2 l_{0}+1}$ and $l_{0}=\left[4 N c / \alpha \log \left(e^{c / 2} / 2\right)\right]+1$.

Since the number of all combinations of $P \in \boldsymbol{A}^{(p, d)}$ and $Q \in \boldsymbol{B}^{(p, d)}$ does not exceed $\left(2 e^{d c}\right)^{2 N}$, we have

$$
\sum_{\substack{P \in \boldsymbol{A}^{(p, d)} \\ Q \in \boldsymbol{B}^{(p, d)}}} P\left(\bigcap_{\substack{X \in \boldsymbol{A}^{(p, d-1)} \\ Y \in \boldsymbol{B}^{(p, d-1)}}} F^{(d-1)}(X, Y) \cap F^{(d)}(P, Q)\right) \leqq 2^{N} \cdot c_{9} e^{-2 d N c} \cdot P(E(A, B)) .
$$

By (5. 5), (5.6) and (5. 7) we have

$$
\begin{aligned}
& P\left(\underset{P \in A^{(p, d)}}{\cup} F^{(d)}(P, Q)\right) \leqq 2^{3 N+1} c_{9} \cdot P(E(A, B)) \cdot\left(\sum_{j=0}^{\infty} e^{-2 j N c}\right) \\
& Q \in B^{(p, d)}
\end{aligned}
$$


Thus it completes the proof if we set a constant $c_{5}$ by $c_{5}=2^{3 N+1} \cdot c_{9} /\left(1-e^{-2 N c}\right)$ in statement of Lemma 9.

Lemma 2, [2. 1] follows from these preparations.

Proof of Lemma 2, [2. 1]. Continuity of the Gaussian process implies that for $A \in \boldsymbol{A}^{(p)}$ and $B \in \boldsymbol{B}^{(p)}, A=B+L\left(L \in \boldsymbol{L}^{(p)}\right)$

$$
\tilde{E}(A, B) \subseteq \bigcup_{h=1}^{\infty} \bigcup_{d=h}^{\infty}\left\{\bigcup_{\substack{P \in A^{(p, d)} \\ Q \in B^{(p, d)}}} F^{(d)}(P, Q)\right\} .
$$

Hence

$$
\begin{aligned}
P(\widetilde{E}(A, B)) & \leqq \lim _{d \rightarrow \infty} P(\underbrace{\bigcup}_{\substack{P \in \boldsymbol{A}^{(p, d)} \\
Q \in \boldsymbol{B}^{(p, d)}}} F^{(d)}(P, Q)) \\
& \leqq c_{5} P(E(A, B)) .
\end{aligned}
$$

Let the pair $(A, B)$ runs over $\boldsymbol{A}^{(p)}$ and $\boldsymbol{B}^{(p)}$, and corresponds the numbering of $E(A, B)$ or $\tilde{E}(A, B)$ to each pair $(A, B)$. Then from the assumption of Lemma 2, [2. 1] and Lemma 7 we have

$$
\sum_{n=1}^{\infty} P\left(\widetilde{E}_{n}\right)<\infty
$$

which proved the lemma.

Proof of Lemma 2, [2. 2]. We use the similar estimates as employed in Lemma 7. Set the number corresponding to $P_{0}=\left[2 e^{3 / \alpha} / \alpha\right]+1$ by $n_{0}$. For proof it is enough to show that if $I(\varphi)=\infty$, then $\sum_{n=n_{0}}^{\infty} P\left(E_{n}\right)=\infty$. Relying on Lemma 3 and Lemma 5, (b), we underestimate $P\left(E_{n}\right)$ as follows:

$$
\begin{aligned}
& \sum_{n=n_{0}}^{\infty} P\left(E_{n}\right)=\sum_{\substack{E(A, B) \in \mathscr{C} \\
\text { for } p \geqq p_{0}}} P(E(A, B)) \\
\geqq & \frac{1}{2^{N+1} \sqrt{2 \pi}} \cdot \sum_{p=p_{0}}^{\infty}\left\{2^{p_{N}}\left(\frac{1}{3} p^{1 / \alpha}\right)^{N} \exp \left(-\frac{1}{2} \varphi^{2}\left(3 v_{p}\right)\right) / \varphi\left(3 v_{p}\right)\right\} \\
\geqq & \frac{1}{2^{N+1} \sqrt{2 \pi}} \cdot \sum_{p=p_{0}}^{\infty}\left\{\left(3 v_{p}\right)^{N-1}\left(3 v_{p+1}-3 v_{p}\right)\left(p^{1 / \alpha} / 3\right)^{2 N} \cdot\right. \\
\geqq & \frac{c_{3}^{-2 N / \alpha}}{2^{N+1} 3^{2 N} \sqrt{2 \pi}} \sum_{p=p_{0}}^{\infty} \int_{3 v_{p}}^{3 v_{p+1}}\left(3 v_{p}\right)^{N-1} K\left[\varphi\left(3 v_{p}\right)\right] d t
\end{aligned}
$$




$$
\geqq \frac{c_{3}^{-2 N / \alpha}}{2^{N+1} 3^{2 N} \sqrt{2 \pi}}\left(\frac{1}{2}\left(\frac{2}{3}\right)^{1 / \alpha}\right)^{N-1} \cdot \sum_{p=p_{0}}^{\infty} \int_{3 v_{p}}^{3 v_{p+1}} t^{N-1} K[\varphi(t)] d t .
$$

Thus we obtain

$$
\sum_{n=n_{0}}^{\infty} P\left(E_{n}\right) \geqq \frac{c_{3}^{-2 N / \alpha}}{2^{N+1} 3^{2 N} \sqrt{2 \pi}}\left(\frac{1}{2}\left(\frac{1}{3}\right)^{1 / \alpha}\right)^{N-1} \cdot\left\{I(\varphi)-\int_{e}^{3 v_{p_{0}}} t^{N-1} K[\varphi(t)] d t\right\} .
$$

which verifies Lemma 2, [2. 2].

Proof of Lemma 2, [2. 3]. We recognize easily that it is sufficient to prove only for the subsequence $\left\{m_{k}\right\}$ such that $\rho\left(u_{n}, u_{m_{k}}\right) \geqq 0$. Using the inequality $(a+b)^{\alpha} \leqq a^{\alpha}+b^{\alpha},(0<\alpha \leqq 1, a>0, b>0) \quad$ (Hardy-Littlewood-Pólya [7]), we have

$$
0 \leqq \rho\left(u_{n}, u_{m_{k}}\right) \leqq d\left(A_{m_{k}}, B_{m_{k}}\right)^{\alpha / 2} / d\left(A_{n}, B_{n}\right)^{x / 2} \longrightarrow 0, m_{k} \longrightarrow \infty
$$

which, for each $n$, implies Lemma 2, [2. 3].

The following lemmas are prepared to refer in the proof of Lemma 2, [2. 4]. For the function $\varphi(t)$ and each $E_{j}$, we define $\boldsymbol{E}_{j}$ by

$$
\boldsymbol{E}_{j}=\left\{E_{n} ; \rho\left(u_{j}, u_{n}\right) \geqq 1 /\left\{\varphi\left(s_{j}\right) \cdot \varphi\left(s_{n}\right)\right\}, n>j\right\},
$$

where $s_{k}$ denotes $1 / d\left(A_{k}, B_{k}\right)$ corresponding to $u_{k}=\left(x\left(A_{k}\right)-x\left(B_{k}\right)\right) / \sigma\left(A_{k}, B_{k}\right)$. Then we have

Lemma 10. $\boldsymbol{E}_{j}$ is a finite set for each $j$.

Proof. From (5. 8), we find

$$
1 /\left\{\varphi^{2}\left(s_{j}\right) \cdot \varphi^{2}\left(s_{n}\right)\right\} \leqq \rho^{2}\left(u_{j}, u_{n}\right) \leqq d^{\alpha}\left(A_{n}, B_{n}\right) / d^{\alpha}\left(A_{j}, B_{j}\right) .
$$

By Lemma 4, (c), the above becomes as follows in terms of $p$ and $p^{\prime}$ corresponding to $E_{j}$ and $E_{n}$, respectively;

$$
\left(c_{3}^{2} p p^{\prime}\right)^{-1} \leqq \rho^{2}\left(u_{j}, u_{n}\right) \leqq\left\{3 v_{p} / p p^{\prime}\right\}^{\alpha},
$$

i.e.

$$
\left(c_{3}^{2} p p^{\prime}\right)^{-1} \leqq 3^{\alpha} \cdot\left(\frac{p^{\prime}}{p}\right) / 2^{\alpha\left(p^{\prime}-p\right)} .
$$

This yields

$$
p^{\prime} \leqq p+c_{10} \cdot \log p,
$$

where

$$
c_{10}=2 \log 2+\left\{\log \left(c_{3}^{2} 3^{\alpha}\right) / \alpha(\log 2)^{2}\right\} .
$$

This assures the lemma. 
By Lemma 10, we can write down for each $j$

$$
\boldsymbol{E}_{j}=\left\{E_{j 1}, E_{j 2}, \cdots, E_{j s(j)}\right\}, j i>j .
$$

Lemma 11 (K.L.Chung, P.Erdös and T. Sirao [3]). Let $U$ and $V$ be two random variables whose joint distribution is a two-dimensional Gaussian distribution and each of them is subjected to one-dimensional standard Gaussian distribution.

Then,

(a) If $\rho(U, V)<1 / a b$, there exists a positive absolute constant $d_{1}$ such that

$$
P(U>a, V>b) \leqq d_{1} P(U>a) \cdot P(V>b) .
$$

(b) There exists two positive absolute constants $d_{2}$ and $\delta$ such that for any $a>0$,

$$
P(U>a, V>a) \leqq d_{2} \cdot \exp \left\{-\delta\left(1-\rho^{2}(U, V) a^{2}\right)\right\} \cdot P(U>a) .
$$

For the proof of (3.1) in Lemma 2, [2. 4] it is convenient to separate

$$
\sum_{i=1}^{s(j)} P\left(E_{j} \cap E_{j i}\right)=\sum_{(1)} P\left(E_{j} \cap E_{j i}\right)+\sum_{(2)} P\left(E_{j} \cap E_{j i}\right),
$$

where $\Sigma_{(1)}$ and $\Sigma_{(2)}$ denote summation over $i$ satisfying

$$
\rho\left(u_{j}, u_{j i}\right)>\left(1-\frac{1}{\sqrt{p}}\right)^{1 / 2} \text { and } \rho\left(u_{j}, u_{j i}\right) \leqq\left(1-\frac{1}{\sqrt{p}}\right)^{1 / 2},
$$

respectively. For each summation we evaluate the summand and the number appearing in the sum.

LeMmA 12. There exists an absolute constant $c_{11}$ such that

$$
\sum_{(1)} P\left(E_{j} \cap E_{j i}\right) \leqq c_{11} \cdot P\left(E_{j}\right) .
$$

Proof. First we estimate $P\left(E_{j} \cap E_{j i}\right)$. From the definition of $\Sigma_{(1)}$, we find a positive integer $k$ such that $k \leqq \sqrt{p}$ and

$$
\left(1-\frac{k}{p}\right)^{1 / 2} \leqq \rho\left(u_{j}, u_{j i}\right)<\left(1-\frac{(k-1)}{p}\right)^{1 / 2} .
$$

Hence, by Lemma 11, (b) and Lemma 4, (c) we obtained

$$
\begin{aligned}
P\left(E_{j} \cap E_{j i}\right) & \leqq d_{2} \exp \left\{-\delta\left(c_{1}^{2} 2 N \log 2\right) p \cdot(k-1) / p\right\} \cdot P\left(E_{j}\right) \\
& \leqq c_{12} \exp \left\{-\delta\left(c_{1}^{2} 2 N \log 2\right) \cdot k\right\} \cdot P\left(E_{j}\right),
\end{aligned}
$$

where

$$
c_{12}=d_{2} \cdot \exp \left\{\delta\left(c_{1}^{2} 2 N \log 2\right)\right\} \text {. }
$$


Next we estimate the number of summand in $\Sigma_{(1)}$. If we apply (5.8) to the present case, we have

$$
\left(1-\frac{k}{p}\right) \cdot d^{\alpha}(A, B) \leqq d^{\alpha}\left(A^{\prime}, B^{\prime}\right) \leqq d^{\alpha}(A, B),
$$

where pairs of points $(A, B)$ and $\left(A^{\prime}, B^{\prime}\right)$ correspond to $E_{j}$ and $E_{j i}$, respectively. Further using (5.9) to estimate the denominator of $\rho\left(u_{j}, u_{f i}\right)$, we have

$$
\left(1-\frac{2 k}{p}\right) d^{\alpha}(A, B) \leqq d^{\alpha}\left(A, B^{\prime}\right)-d^{\alpha}\left(B, B^{\prime}\right),
$$

$$
\left(1-\frac{2 k}{p}\right) \cdot d^{\alpha}(A, B) \leqq d^{\alpha}\left(A^{\prime}, B\right)-d^{\alpha}\left(A, A^{\prime}\right) .
$$

Thus the number in $\Sigma_{(1)}$ does not exceed the number of point $A^{\prime}$ and $B^{\prime}$ which are contained in the region determined by (5.9) and (5.10). A computation shows that this region has volume $V$ smaller than $\left\{2(4 k)^{1 / \alpha} / 2^{p}\right\}^{N}$. On the other hand, $p^{\prime}$ is estimated by $p^{\prime} \leqq p+l_{0}$, where $l_{0}$ is an absolute constant integer. In fact, if we set $l_{0}, n_{0}$ and $m_{0}$ by $l_{0}=4 \bigvee n_{0} \vee m_{0}$ where $n_{0}=\min \left\{n ; 2\left(1-(3 / 16)^{\alpha}\right)-\sqrt{2} \geqq n \cdot 3^{\alpha} / 2^{n \alpha}\right\}$ and $m_{0}=\max \left\{n ; n+2 \geqq 2^{n \alpha+1} / 3^{\alpha}\right\}$, respectively. If we consider (5.9) in term of $p$ and $p^{\prime}$, we see that $n_{0}$ and $m_{0}$ are minimum integers to break inequalities in (5.10). Since the above argument permits us to overestimate the number in $\Sigma_{(1)}$ as

$$
V /\left(1 / 2^{p+l_{0}}\right)^{N} \leqq\left(2^{l_{0}+1} \cdot 4^{1 / \alpha}\right)^{N} k^{N / \alpha},
$$

we have

$$
\sum_{(1)} P\left(E_{j} \cap E_{j i}\right) \leqq c_{12}\left(2^{l_{0}+1} \cdot 4^{1 / \alpha}\right)^{N} P\left(E_{j}\right) \cdot \sum_{k=1}^{\infty} k^{N / 2} \cdot \exp \left\{-\delta\left(c_{1}^{2} 2 N \log 2\right) k\right\},
$$

which completes the proof if we set $c_{11}$ by the coefficient of $P\left(E_{j}\right)$ in the right-hand side.

Lemma 13. There exists an absolute constant $c^{\prime}{ }_{11}$ such that

$$
\Sigma_{(2)} P\left(E_{j} \cap E_{j i}\right) \leqq c^{\prime}{ }_{11} \cdot P\left(E_{j}\right) .
$$

Proof. First we estimate the summand and next the number in $\Sigma_{(2)}$. Since we have $s_{j}>s_{j i}$ for $j i>j\left(s_{k}=1 / d\left(A_{k}, B_{k}\right)\right)$, it holds by Lemma 4, (c) and Lemma 11, (b)

$$
P\left(E_{j} \cap E_{j i}\right) \leqq P\left(u_{j}>\varphi\left(s_{j}\right), u_{j i}>\varphi\left(s_{j}\right)\right)
$$




$$
\begin{aligned}
& \leqq d_{2} \exp \left\{-\delta\left(1-\rho^{2}\left(u_{j}, u_{j i}\right) \cdot \varphi\left(s_{j}\right)\right\} \cdot P\left(u_{j}>\varphi\left(s_{j}\right)\right)\right. \\
& \leqq d_{2} \exp \left(-\delta c_{3} \sqrt{p}\right) \cdot P\left(E_{j}\right)
\end{aligned}
$$

Set

$$
\begin{aligned}
& l_{0}^{\prime}=m_{0}^{\prime} \vee n_{0}^{\prime} \vee 2, \\
& m_{0}^{\prime}=\min \left\{l ; 2\left(e+c_{10}\right)>e \cdot 2^{l}\right\}, \\
& n_{0}^{\prime}=\min \left\{l ; \log \left(1 / c_{10}\right)<l\left(\frac{1}{\alpha}-1\right)\right\} .
\end{aligned}
$$

For $(A, B)$ corresponding to $E_{j}$ and $\left(A^{\prime}, B^{\prime}\right)$ corresponding to $E_{j i}$, if we have inequality, $d\left(A^{\prime}, B\right)$ or $d\left(A, B^{\prime}\right) \geqq\left(p^{l_{0}^{\prime} / \alpha}\right) / 2^{p}$, then $\rho\left(u_{j}, u_{j i}\right)$ does not satisfy the condition of $\boldsymbol{E}_{j}$. This implies that for all $E_{j i} \in \boldsymbol{E}_{j}, d\left(A^{\prime}, B\right)$ and $d\left(A, B^{\prime}\right) \leqq\left(p^{l^{\prime}} / \alpha\right) / 2^{p}$. Since $A^{\prime}$ and $B^{\prime}$ are contained in a cube with volume $\left(2 p \nu_{0}^{\prime} / 2^{p}\right)^{N}$, the number \# of such points $A^{\prime}$ or $B^{\prime}$ is dominated as

$$
\begin{gathered}
\# \leqq\left(2 p^{l_{0}^{\prime} / \alpha} / 2^{p}\right) /\left(1 / 2 p^{\prime}\right)^{N} \\
\leqq 2^{N} \cdot p^{N\left(\frac{l_{0}^{\prime}}{\alpha}+c_{10} \log 2\right)}
\end{gathered}
$$

Thus we have estimate of $\Sigma_{(2)}$;

$$
\Sigma_{(2)} P\left(E_{j} \cap E_{j i}\right) \leqq d_{2} 2^{2 N} p^{2 N}\left(\frac{l_{0}^{\prime}}{a}+c_{10} \log 2\right) \cdot \exp \left\{-\delta c_{3} \sqrt{p}\right\} \cdot P\left(E_{j}\right),
$$

which verifies the lemma if we set $c^{\prime}{ }_{11}$ by

$$
c^{\prime}{ }_{11}=\left(\delta \cdot c_{3}\right)^{M} \cdot M ! / 2 \text { and } M=\left\{N\left(\frac{l_{0}^{\prime}}{\alpha}+c_{10} \log 2\right)\right\}+1 .
$$

Proof of Lemma 2, [2. 4]. If we set $k_{1}=c_{11} \vee c^{\prime}{ }_{11}$, it yields (3. 1). From Lemma 11, (a) we find for $E_{k} \notin \boldsymbol{E}_{j},(k>j)$,

$$
P\left(u_{j}>\varphi\left(s_{j}\right), u_{k}>\varphi\left(s_{k}\right)\right)<d_{1} \cdot P\left(u_{j}>\varphi\left(s_{j}\right)\right) \cdot P\left(u_{k}>\varphi\left(s_{k}\right)\right),
$$

since $\rho\left(u_{j}, u_{k}\right)<1 /\left\{\varphi\left(s_{j}\right) \cdot \varphi\left(s_{k}\right)\right\}$. This implies (3.2).

We established the proof of theorem completely.

\section{REFERENCES}

[1] Yu.K. Belayev, Continuity and Hölder Conditions for sample functions of stationary Gaussian processes. Proc. 4-th Berkeley symposium Math. Stat. and Probability. (1961) 23-33.

[2] K.L.Chung and P.Erdös, On the application of the Borel-Cantelli lemma. Trans. Amer.Math. Soc. Vol. 72, (1952) 179-186. 
[ 3 ] K.L. Chung, P. Erdös and T. Sirao, On the Lipschitz's condition for Brownian motion. Jour. Math. Soc. Japan. Vol. 16, (1960) 263-274.

[4] R.M. Dudley, The size of compact subsets of Hilbert space and continuity of Gaussian processes. Jour. Functional Analysis. Vol. 1, No. 3, (1967) 290-330.

[ 5 ] X. Fernique, Continuité des Processus Gaussien. C.R. t.258, (1964) Groupe 1, 6058-6060.

[6] R. Gangolli, Positive definite kernels on homogeneous spaces and certain stochastic processes related to Lévy's Brownian motion of several parameters. Ann. Inst. Henri Poincaré. Vol. 3, (1967) Section B, 121-225.

[ 7 ] Hardy-Littlewood-Pólya, Inequalities. 2-nd, edit. 1964.

[ 8 ] P. Lévy, Theorie de l'addition des variables aléatoires. 1937.

[9] P. Lévy, Processus stochastique et movement brownien. 1948.

[10] I. Petrovsky, Zur ersten Randwertaufgabe der Wärmeleitungsgleichung. Composito Math. 1. (1935) 383-419.

[11] T. Sirao, On the continuity of Brownian motion with a multidimensional parameter. Nagoya Math. Jour, Vol. 16, (1960) 135-156.

[12] T. Sirao and H. Watanabe, On the Hölder continuity of stationary Gaussian processes. Proc. Japan Acad. Vol. 44, No. 6 (1968) 482-484.

Kobe College of Commerce. 